This paper investigates the vibration characteristics of the coupling system of a micro scale fluid-loaded rectangular isotropic plate attached with a uniformly distributed mass. Previous literature has respectively studied the changes on the plate vibration induced by an acoustic field or by the attached mass loading. This paper investigates the issue of involving these two types of loading simultaneously. Based on Lamb's assumption of the fluid-loaded structure and the Rayleigh-Ritz energy method, this paper presents an analytical solution for the natural frequencies and mode shapes of the coupling system. Numerical results for micro plates with different types of boundary conditions have also been obtained and compared with experimental and numerical results from previous literature. The theoretical model and novel analytical solution are of particular interest in the design of microplate based biosensing devices.

Keywords: microplate dynamics, fluid interaction, micro biosensing system 


\title{
Vibration analysis of submerged micro rectangular plates with distributed mass loading
}

\author{
By Zhangming WU ${ }^{1}$, Xianghong MA ${ }^{1}$, \\ Peter N Brett ${ }^{1}$ And Jinwu Xu ${ }^{2}$ \\ ${ }^{1}$ School of Engineering and Applied Science, \\ Aston University, UK \\ ${ }^{2}$ Dept of Mechanical Engineering, \\ University of Science and Technology Beijing, China
}

\section{Introduction}

Microplate dynamics in fluid is an important research field in the design of bioMEMS. This is due to the fact that most microstructure based biosensors need to interact with biological particles in a natural fluid environment. This paper presents an analytical method to solve the dynamics of micro rectangular plates in fluid. The novel method also takes into consideration distributive mass loading, which represents the effect of adhesion of biological cells and particles to the surface of the biosensing plate. The analytical method can be applied widely in the modelling and design of microstructure based biosensing components.

Interest in the study of the vibration of thin plates contacting with fluid started with the work of Lamb(Lamb, 1921), who presented a simple model and calculated resonant frequencies of a thin circular plate in contact with water. Lamb's model assumes the fluid to be inviscid, incompressible and to have no effect on the vibration mode shapes of the plate. The concept of added mass was first proposed to determine the resonant frequencies with fluid mass loading on the plate. Subsequently, other work analysed vibrating response for submerged or floating plates with varied geometries and boundary conditions based on Lamb's assumption and the added mass method(Greenspon, 1961; Lindholm et al., 1965; Meyerhoff, 1970). More recently Kwak and Kim(Kwak, 1991, 1994, 1996) have presented a computational method for circular or rectangular plates in water using non-dimensional added virtual mass incremental (NAVMI) factors. Here the NAVMI factors are predicted by the ratio of the kinetic energies of the fluid and plate respectively. Liang et al.(Liang et al., 2001) used an empirical added mass formulation and Rayleigh-Ritz method to analyse the vibrational frequencies and mode shape of submerged cantilever plates. Ayela and Nicu(Nicu and Ayela, 2007) proved experimentally that the Lamb's theoretical model for plates vibrating in fluid is valid for microscale structures. In these papers the added mass method was widely employed to approximately evaluate natural frequencies of fluid-loaded plates and other structures. Finding a general analytical solution for rectangular plate dynamics in fluid remains a challenge. 
The difficulty in obtaining analytical solutions to added mass or fluid loading effects on plate dynamics is due principally to the integral singularity of acoustic impedance(Chang and Leehey, 1996). Recently useful explicit integration methods have been developed for evaluating the acoustic impedance in special cases. Graham(Graham, 1995, 2007) presented an asymptotic analytical solution for simply supported plates using contour integration to replace the impedance in double Fourier transform. Pierce (Pierce et al., 2002) eliminated the integral singularity by reducing the double integrals to single integrals and transforming Cartesian coordinates into polar coordinates. While Pierce's approach was applied only to special cases, where each impedence integral is of finite sum with regard to the exponential function, it can be applied more generally. In this paper Pierce's method is applied in the evaluation of kinetic energy in the fluid. This can be used to represent the effect of incompressible fluid loading and is analogous to acoustic impedance.

Most published literature on the vibration of mass loaded plates is focused on concentrated masses. Few papers study the effects of distributed mass in vacuo. Kopmaz and Telli(Kopmaz and Telli, 2002) presented a mathematical model of a rectangular plate carrying a uniformly distributed mass using Galerkin's method to discretize the partial differential equations of the plate. Wong(Wong, 2002) proposed a formulation to the eigenvalue problem of plates with distributed mass loading based on the Rayleigh-Ritz energy variational principle. This paper investigates the effects of both distributed mass loading and fluid interaction on the dynamics of microplates under different boundary conditions. It is the first presentation of an analytical solution to this typical dynamical problem of microstructures.

\section{Theoretical model of rectangular plates}

In most theoretical analyses of the dynamics of a plate in the fluid, a simplified model of the plate placed in an infinite plane baffle is usually considered. This is also applicable to the case of a microplate as it is always fabricated on a wafer substrate with the size of the wafer typically 1000 times that of the thin micro plate. The schematic diagram of the sensing microplate model is shown in Figure 1. The top surfuce of microplate is used as the sensing surface with the biological particles attached, and herein the microplate is immersed in fluid with both sides contact with fluid. The sensing microplate is assumed thin, elastic, isotropic and surrounded by infinite rigid baffles. A small circular distributed mass resting on the plate sensing surface is used to simulate attached biological particles.

\section{Insert Figure 1}

Assuming that the distributed mass can be considered fixed to the plate such that no additional strain energy is induced(Wong, 2002), and ignoring effects of rotatory inertia and transverse shear deformation, the governing equation of the forced vibration of the rectangular isotropic plate can be written in the following form.

$$
D \nabla^{4} w(x, y, t)+\rho_{p} h \frac{\partial^{2} w(x, y, t)}{\partial t^{2}}+\bar{m}_{c} \mathcal{H}(x, y) \frac{\partial^{2} w(x, y, t)}{\partial t^{2}}=p(x, y, t)
$$


Where $w(x, y, t)$ is the transverse deflection function of the plate, $\rho_{p}$ and $h$ are the volume density and thickness of plate respectively, $D=E h^{3} / 12\left(1-\nu^{2}\right)$ is the flexural rigidity, $E$ is Young's modulus, $\nu$ is Poisson's ratio, $p(x, y, t)$ is the acoustic pressure on the plate surface and $\bar{m}_{c}$ is the mass per unit area of cells present on the plate. The Heaviside function $\mathcal{H}(x, y)$ is used to describe the circular domain of the distributed mass on plate and can be expressed in the following form, where $\left(x_{c}, y_{c}\right)$ is the location of the centre of the distributed mass and $R$ is the radius of the distributed mass.

$$
\begin{aligned}
\mathcal{H}(x, y)= & \left\{H\left[x-\left(x_{c}-R\right)\right]-H\left[x-\left(x_{c}+R\right)\right]\right\} . \\
& \left\{H\left[y-\left(y_{c}-\sqrt{R^{2}-x^{2}}\right)\right]-H\left[y-\left(y_{c}+\sqrt{R^{2}-x^{2}}\right)\right]\right\}
\end{aligned}
$$

The linear solution of Eq.(2.1) can be obtained by summing a series of eigenfunctions in each separated mode(Szilard, 1974).

$$
w(x, y, t)=\sum_{m=1}^{\infty} \sum_{n=1}^{\infty} W_{m n} X_{m}(x) Y_{n}(y) \cdot \theta(t)
$$

Where $\theta(t)$ is the time dependency of the transverse displacements, in a harmonic vibration $\theta(t)=\sin (\omega t+\vartheta)$ and $\vartheta$ is the initial phase difference. For the linear vibration without acoustic pressure $(p(x, y, t)=0)$ and mass attachment $\left(\bar{m}_{c}=0\right)$, $X_{m}(x)$ and $Y_{n}(y)$ are the orthogonal mode shape functions that satisfy the boundary conditions in $x$ and $y$ directions, respectively. From Leissa's analysis(Leissa, 1969), $X_{m}(x)$ and $Y_{n}(y)$ can be chosen as the same mode shape functions of beams that have the same boundary conditions. Three types of plate boundary conditions have been investigated in this paper: all clamped(C-C-C-C), cantilever (C-F-F-F) and the case of two opposite edges free with the other two clamped(C-F-C-F). Correspondingly, three types of beam function are required as follows:

(a)For clamped-clamped(C-C) boundary conditions,

$$
\begin{aligned}
X_{m}(x)= & \cosh \left(\frac{\epsilon_{m} x}{L_{a}}\right)-\cos \left(\frac{\epsilon_{m} x}{L_{a}}\right)-\alpha_{m}\left[\sinh \left(\frac{\epsilon_{m} x}{L_{a}}\right)-\sin \left(\frac{\epsilon_{m} x}{L_{a}}\right)\right] \\
& \cos \left(\epsilon_{m}\right) \cosh \left(\epsilon_{m}\right)-1=0, \quad \alpha_{m}=\frac{\cosh \left(\epsilon_{m}\right)-\cos \left(\epsilon_{m}\right)}{\sinh \left(\epsilon_{m}\right)-\sin \left(\epsilon_{m}\right)}
\end{aligned}
$$

(b)The beam function of clamped-free $(\mathrm{C}-\mathrm{F})$ boundary conditions has the same form as clamped-clamped, with different constants of $\epsilon_{m}$ and $\alpha_{m}$,

$$
\cos \left(\epsilon_{m}\right) \cosh \left(\epsilon_{m}\right)+1=0, \quad \alpha_{m}=\frac{\sinh \left(\epsilon_{m}\right)-\sin \left(\epsilon_{m}\right)}{\cosh \left(\epsilon_{m}\right)-\cos \left(\epsilon_{m}\right)}
$$

(c)For free-free(F-F) boundary conditions,

$$
X_{1}(x)=1 \quad X_{2}(x)=\sqrt{3}(2 x-1)
$$

$$
X_{m}(x)=\mu_{m}\left[\cosh \left(\frac{\epsilon_{m} x}{L_{a}}\right)+\cos \left(\frac{\epsilon_{m} x}{L_{a}}\right)\right]-\alpha_{m}\left[\sinh \left(\frac{\epsilon_{m} x}{L_{a}}\right)+\sin \left(\frac{\epsilon_{m} x}{L_{a}}\right)\right]
$$




$$
\text { where } \mu_{m}=\frac{\cosh \left(\epsilon_{m}\right)-\cos \left(\epsilon_{m}\right)}{\sinh \left(\epsilon_{m}\right) \sin \left(\epsilon_{m}\right)} \quad \text { and } \quad \alpha_{m}=\frac{\sinh \left(\epsilon_{m}\right)+\sin \left(\epsilon_{m}\right)}{\sinh \left(\epsilon_{m}\right) \sin \left(\epsilon_{m}\right)}
$$

In this case the constant of $\epsilon_{m}$ for free-free boundary conditions is the same as that for the clamped-clamped conditions. $Y_{n}(y)$ is in the same form, replacing $x$ for $y$ and $L_{a}$ for $L_{b}$ within each beam shape function respectively.

\section{The effects of fluid loading on plate}

Many published articles in the literature show that plate vibrational characteristics are sensitive to heavy fluid loading. Eq. (2.3) shows that a vibrating plate in vacuo has infinite discrete orthogonal modes. In the presence of fluid, each mode of the plate motion can generate an acoustic pressure disturbation. Therefore the total external force applied on the plate is the sum of the excitation force and the resultant acoustic pressure field. Nevertheless in general the resultant acoustic pressure induced by a single in vacuo mode is not orthogonal to the other modes(Junger and Feit, 1972) and will excite other modes. This implies that one in vacuo mode can "indirectly apply" forces and transfer energy to another mode via the coupling fluid medium(Lax, 1944). This cross-modal coupling phenomenon causes two principal effects on the behavior of plate vibration: (a)The fluid generates additional inertia loading on the plate, and will lower the plate resonant frequencies. (b)The vibrating plate radiates acoustic energy into the fluid, this dissipation of the plate vibrational energy adds a resistive term to the impedances of the plate(Lax, 1944), and as a result the Q-factor of the vibrating plate will decrease.

The acoustic velocity field induced by plate motion is governed by the threedimensional Helmholtz wave equation(Junger and Feit, 1972),

$$
\nabla^{2} \Phi(x, y, z, t)=\frac{1}{c^{2}} \frac{\partial^{2} \Phi(x, y, z, t)}{\partial t^{2}}
$$

where $\nabla^{2}$ is the three-dimensional Laplacian operator, $c$ is the velocity of sound within fluid and $\Phi(x, y, z, t)$ is the velocity potential of the fluid. Boundary conditions(continuity of normal velocity) at the interface between the fluid and the baffled $\operatorname{plate}(z=0)$ can be expressed as: $\partial \Phi /\left.\partial z\right|_{z=0}=\partial w / \partial t$ on the plate, and $\partial \Phi /\left.\partial z\right|_{z=0}=0$ on the baffle plane.

For a steady-state response in harmonic motion, the acoustic velocity potential becomes $\Phi(x, y, z, t)=\phi(x, y, z) e^{-i \omega t}$. One form of the solution of the Helmholtz wave Eq.(3.1) is given by the Rayleigh's formula(Junger and Feit, 1972):

$$
\phi(x, y, z)=\frac{i \omega}{2 \pi} \iint_{S} \frac{w(\xi, \eta) \exp \left(i k \sqrt{(x-\xi)^{2}+(y-\eta)^{2}+z^{2}}\right)}{\sqrt{(x-\xi)^{2}+(y-\eta)^{2}+z^{2}}} d \xi d \eta
$$

where $\phi(x, y, z)$ is the spatial velocity potential function. $k=\omega / c$ is the acoustic wave number and $S$ is the integral domain for the whole rectangular plate.

When dealing with the vibration analysis of low modes of the plate, we can make the following assumptions and simplifications for the fluid(Kwak, 1991): (a)The 
fluid is incompressible and inviscid. In general water is ideal and satisfies the "incompressible" assumption. Therefore the Helmholtz wave equation reduces to a single Laplace transform $(c \rightarrow \infty, k \rightarrow 0)$. The influence of fluid viscosity on the vibration of plate is very small, and has been verified by Atkinsona et al.(Atkinsona and de Lara, 2007) and Nicu(Nicu and Ayela, 2007). (b)The fluid is infinite, irrotational and has no wall effects. Thus the primary effect of the surrounding fluid on the vibrating plate is the additional inertial. The fluid-induced inertial effect on the plate can be represented by added mass factor $\left(\beta_{m n}\right)$ or $\operatorname{NAVMI}$ factor $\left(\Gamma_{m n}\right)$, which is simply the function of plate natural modes. They are defined as follows

$$
\beta_{m n}=\left(w_{v, m n} / w_{f, m n}\right)^{2}-1=\Gamma_{m n} \frac{\rho_{f} \varepsilon L_{b}}{\rho_{p} h}
$$

where $w_{v, m n}$ and $w_{f, m n}$ are the natural frequencies of plate in vacuo and in fluid respectively, $\rho_{f}$ is the density of fluid, $\varepsilon$ is the plate aspect ration $L_{b} / L_{a}$. From the view of energy, the added mass effect can be evaluated as proportional to the kinetic energies of the fluid $\left(T_{f}\right)$, which is defined as(Kwak, 1991)

$$
T_{f}=-\left.\frac{1}{2} \rho_{f} \int_{-\infty}^{\infty} \int_{-\infty}^{\infty} \frac{\partial \phi(x, y, z)}{\partial z}\right|_{z=0} \phi(x, y, 0) d x d y
$$

Substituting Eq.(3.2) into Eq.(3.4) with the case $k=0$ and combining the fluid-structure interface boundary conditions, we can rewrite $T_{f}$ in the form

$$
T_{f}=\frac{\rho_{f} \omega^{2}}{4 \pi} \iint_{S} \iint_{S} \frac{w(x, y) w(\xi, \eta)}{\sqrt{(x-\xi)^{2}+(y-\eta)^{2}}} d x d y d \xi d \eta
$$

Here $T_{f}$ only represents the fluid energy of one side. Therefore if the plate is submerged with both sides contacting fluid, the kinetic energy of fluid $T_{f}$ should be doubled for the computation.

\section{Analytic solution using Rayleigh-Ritz method}

Using Rayleigh's quotient(Zhu, 1994), the natural frequencies of plate in vacuo can be approximately determined by the ratio between the maximum potential $\operatorname{energy}\left(U_{p}\right)$ and the reference kinetic energy $\left(T_{p}^{*}\right)$ of the plate. When dealing with the coupling problem of a fluid-loaded plate with mass attachment, the reference kinetic energy should be replaced by summing that of the plate, the fluid $\left(T_{f}^{*}\right)$ and the $\operatorname{mass}\left(T_{m}^{*}\right)$. This relationship can be written as

$$
\omega_{c}^{2}=\frac{U_{p}}{T_{p}^{*}+T_{f}^{*}+T_{m}^{*}}
$$

where $\omega_{c}$ is the natural frequency of the plate in fluid with mass attached. The potential energy and reference kinetic energy of the plate are defined by Eqs. (4.2) and (4.3).

$$
U_{p}=\frac{D}{2} \iint_{S}\left\{\left(\frac{\partial^{2} w}{\partial x^{2}}+\frac{\partial^{2} w}{\partial y^{2}}\right)^{2}-2(1-\nu)\left[\frac{\partial^{2} w}{\partial x^{2}} \frac{\partial^{2} w}{\partial y^{2}}-\left(\frac{\partial^{2} w}{\partial x \partial y}\right)^{2}\right]\right\} d x d y
$$




$$
T_{p}^{*}=\frac{1}{2} \rho_{p} h \iint_{S} w^{2}(x, y) d x d y
$$

The reference kinetic energy of fluid $T_{f}^{*}=T_{f} / \omega^{2}$. Same with $T_{f}, T_{f}^{*}$ need to be doubled in the case of fully submerged plates.

The reference kinetic energy of the distributed mass can be obtained by employing the aforementioned Heaviside function.

$$
T_{m}^{*}=\frac{1}{2} \iint_{S} \bar{m}_{c} \mathcal{H}(x, y) w^{2}(x, y) d x d y
$$

By assuming that mode shapes of the plate are virtually the same with the plate submerged, then all the above energy formulations can be evaluated using the "dry" plate mode shapes(Eq. (2.4)-Eq.(2.9)). The total energy of the coupled system then can be expressed as

$$
V=U_{p}-\omega^{2}\left(T_{p}^{*}+T_{f}^{*}+T_{m}^{*}\right)
$$

The expanded formula of these energy equations, in terms of the plate orthogonal shape functions, can be obtained by substituting Eq. (2.3) into Eqs. (4.2), (3.5), (4.3), (4.4). A series of eigenfunctions can be obtained by minimizing the Eq. (4.5) with respect to the unknown deflection coefficient $W_{q r}$, such that

$$
\frac{\partial V}{\partial W_{q r}}=0
$$

These eigenfunctions are

$$
\sum_{m=1}^{\infty} \sum_{n=1}^{\infty}\left\{U_{p, m n q r}-\omega^{2}\left(T_{p, m n q r}^{*}+T_{f, m n q r}^{*}+T_{m, m n q r}^{*}\right)\right\} W_{m n}=0
$$

where

$$
\begin{gathered}
U_{p, m n q r}=\frac{D}{2} \iint_{S}\left\{\ddot{X}_{m}(x) \ddot{X}_{q}(x) Y_{n}(y) Y_{r}(y)+X_{m}(x) X_{q}(x) \ddot{Y}_{n}(y) \ddot{Y}_{r}(y)+\right. \\
\left.2 \nu \ddot{X}_{m}(x) X_{q}(x) Y_{n}(y) \ddot{Y}_{r}(y)+2(1-\nu) \dot{X}_{m}(x) \dot{X}_{q}(x) \dot{Y}_{n}(y) \dot{Y}_{r}(y)\right\} d x d y \\
T_{p, m n q r}^{*}=\frac{1}{2} \rho_{p} h \iint_{S} X_{m}(x) X_{q}(x) Y_{n}(y) Y_{r}(y) d x d y \\
T_{f, m n q r}^{*}=\frac{\rho_{f}}{4 \pi} \iint_{S} \iint_{S} \frac{X_{m}(x) Y_{n}(y) X_{q}(\xi) Y_{r}(\eta)}{\sqrt{(x-\xi)^{2}+(y-\eta)^{2}}} d x d y d \xi d \eta \\
T_{m, m n q r}^{*}=\frac{1}{2} \bar{m}_{c} \iint_{S} X_{m}(x) X_{q}(x) Y_{n}(y) Y_{r}(y) \mathcal{H}(x, y) d x d y
\end{gathered}
$$


Alternatively, we can rewrite Eq. (4.7) in matrix form

$$
\left\{[\mathbf{A}]-\omega^{2}[\mathbf{I}]\right\}\{x\}=0
$$

where $\mathbf{A}=\mathbf{M}^{-1} \mathbf{K}$ and $\mathbf{I}$ is the unit matrix. $\mathbf{K}$ and $\mathbf{M}$ are the stiffness matrix and inertial matrix of the vibration system respectively, and their elements are

$$
\begin{gathered}
K_{i j}=U_{p, m n q r} \\
M_{i j}=T_{p, m n q r}^{*}+T_{f, m n q r}^{*}+T_{m, m n q r}^{*} \\
i=l(q-1)+r, \quad j=l(m-1)+n, \quad l \in \mathbb{N}^{+}
\end{gathered}
$$

Hence, the natural frequencies and corresponding mode shapes of the coupled plate can be obtained by extracting the eigenvalues and eigenvectors of matrix $\mathbf{A}$ respectively.

As mentioned before, kinetic energy of fluid $T_{f, m n q r}^{*}$, Eq. (4.10), is an integral with a potential singularity in the denominator and therefore one cannot perform a straightforward numerical evaluation. Following Pierce's integral methodology(Pierce et al., 2002), the fourfold integral of $T_{f, m n q r}^{*}$ can be reduced to a double integral

$$
T_{f, m n q r}^{*}=\frac{\rho_{f}}{4 \pi} L_{a}^{2} L_{b}^{2} \int_{0}^{1} \int_{0}^{1} \frac{1}{\mathcal{R}} F X(u, m, q) F Y(v, n, r) d u d v
$$

where $\mathcal{R}=\sqrt{L_{a}^{2} u^{2}+L_{b}^{2} v^{2}}$ and inner integrand function $F X(u, m, q), F Y(v, n, r)$ are defined as

$$
\begin{gathered}
F X(u, m, q)=\int_{u}^{1} X_{m}\left(x^{\prime}\right) X_{q}\left(x^{\prime}-u\right) d x^{\prime}+\int_{0}^{1-u} X_{m}\left(x^{\prime}\right) X_{q}\left(x^{\prime}+u\right) d x^{\prime} \\
F Y(v, n, r)=\int_{v}^{1} Y_{n}\left(y^{\prime}\right) Y_{r}\left(y^{\prime}-u\right) d y^{\prime}+\int_{0}^{1-v} Y_{n}\left(y^{\prime}\right) Y_{r}\left(y^{\prime}+u\right) d y^{\prime}
\end{gathered}
$$

where $x^{\prime}=x / L_{a}, y^{\prime}=y / L_{b}, \xi^{\prime}=\xi / L_{a}, \eta^{\prime}=\eta / L_{b}$, and $u=x^{\prime}-\xi^{\prime}, v=y^{\prime}-$ $\eta^{\prime}$. Substituting the plate mode shape functions(Eq. (2.4)-Eq.(2.9)) into Eq.(4.14), Eq.(4.15) and decomposing the resulting equations into a series of single integrals, functions $F X(u, m, q)$ and $F Y(v, n, r)$ can be evaluated in closed forms.

In order to eliminate the square root singularity, the whole integral of Eq.(4.13) can be transformed into a polar coordinate system. The square integral domain within the $(u, v)$ plane is divided into two equal and opposite right-angle triangles(Pierce et al., 2002). The leading diagonal of the common hypotenuse of the two triangles is the line from the origin to $(1,1)$. For the lower triangle we can define $u=\mathcal{R} \cos \theta / L_{a}, v=\mathcal{R} \sin \theta / L_{b}$ with the new integral domain $0<\mathcal{R}<$ $L_{a} / \cos (\theta), 0<\theta<\tan ^{-1}\left(L_{b} / L_{a}\right)$. Analogous relationships can be defined for the upper triangle by interchanging $a$ with $b$. The integral of Eq.(4.13) becomes

$$
\begin{array}{r}
T_{f, m n q r}^{*}=\frac{\rho_{f}}{4 \pi} L_{a} L_{b}\left(\int_{0}^{\theta_{1}} \int_{0}^{\frac{L_{a}}{\cos (\theta)}} F X\left(\frac{\mathcal{R} \cos (\theta)}{L_{a}}, m, q\right) F Y\left(\frac{\mathcal{R} \sin (\theta)}{L_{b}}, n, r\right) d \theta d \mathcal{R}\right. \\
\left.+\int_{0}^{\theta_{2}} \int_{0}^{\frac{L_{b}}{\cos (\theta)}} F X\left(\frac{\mathcal{R} \sin (\theta)}{L_{a}}, m, q\right) F Y\left(\frac{\mathcal{R} \cos (\theta)}{L_{b}}, n, r\right) d \theta d \mathcal{R}\right)
\end{array}
$$


where $\theta_{1}=\tan ^{-1}\left(L_{b} / L_{a}\right)$ and $\theta_{2}=\tan ^{-1}\left(L_{a} / L_{b}\right)$

Normalizing the integral domain of $\mathcal{R}$ into $[0,1]$, we can rewrite Eq.(4.16) in the following form

$$
\begin{aligned}
T_{f, m n q r}^{*}=\frac{\rho_{f}}{4 \pi} L_{a} L_{b} & \left(\int_{0}^{1} \int_{0}^{\theta_{1}} F X(\mathcal{R}, m, q) F Y\left(\frac{\mathcal{R} L_{a} \sin (\theta)}{L_{b} \cos (\theta)}, n, r\right) \frac{L_{a}}{\cos (\theta)} d \mathcal{R} d \theta\right. \\
& \left.+\int_{0}^{1} \int_{0}^{\theta_{2}} F X\left(\frac{\mathcal{R} L_{b} \sin (\theta)}{L_{a} \cos (\theta)}, m, q\right) F Y(\mathcal{R}, n, r) \frac{L_{b}}{\cos (\theta)} d \mathcal{R} d \theta\right)
\end{aligned}
$$

Consequently, with the closed-form solution of functions $F X$ and $F Y$ we can accurately evaluate Eq.(4.17) by using normal numerical quadrature routines.

\section{Numerical results}

The resonant frequencies and mode shapes of the submerged microplate carrying a distributed mass are obtained by numerically solving a truncated subset of the infinite series of Eq. (4.7) in MATLAB codes. All of the simulation results presented in this part are generated by using $(25 \times 25)$ terms of Matrix $\mathbf{A}$, and the reference kinetic energy of fluid $\left(T_{f}^{*}\right)$ is doubled for the case of submerged microplate. It can be proved that $(25 \times 25)$ terms are able to result in sufficient convergence. The material properties of the plate, fluid (water) and attached mass used in numerical computation are as follows:

- Plate Length: $L_{a}=100 \mu m$

- Plate Width: $L_{b}=100 \mu m$

- Plate Thickness: $h=5 \mu m$

- Plate Young's Modulus: $E=150 G P a$

- Plate Poisson's Ratio: $\nu=0.17$

- Plate Density: $\rho_{p}=2330 \mathrm{~kg} / \mathrm{m}^{3}$

- Fluid Density: $\rho_{f}=1000 \mathrm{~kg} / \mathrm{m}^{3}$

- Radius of Attached Mass: $R=20 \mu m$

- Surface Mass Density of Attached Mass: $\bar{m}_{c}=0.02 \mathrm{~kg} / \mathrm{m}^{2}$

From Eq.(4.7), it can be seen that when $T_{f}^{*}=0$ and $T_{m}^{*}=0$, the coupled system reduces to the case of unloaded plate motion in vacuo, and if $T_{m}^{*}=0$, the problem becomes the one of an unloaded plate vibrating in a fluid. Figure. 2-4 respectively shows the plate resonant frequencies and mode shapes for three types of boundary conditions in four different loading cases: (A)plate in vacuo without carrying the mass, (B)plate only under fluid loading, (C)involved both fluid-loading and a center mass attachment $\left(x_{c}=L_{a} / 2, y_{c}=L_{b} / 2\right)$, (D)is the case with a corner mass attachment(In Figure 2 and 4 , the corner mass is at $x_{c}=L_{a} / 4, y_{c}=L_{b} / 4$. In 
Figure 3 , the corner mass is at $\left.x_{c}=3 L_{a} / 4, y_{c}=3 L_{b} / 4\right)$.

\section{Insert Figure 2}

\section{Insert Figure 3}

\section{Insert Figure 4}

From these three data plots, we can see that both the fluid loading and the attached distributive biological particles have significant impact on the resonant frequencies and mode shapes of micro plates. The inertial forces of fluid sharply reduce resonant frequencies, especially in the fundamental modes (by more than $50 \%$ ). Fluid has a reduced effect on most of mode shapes, some phase changes can be found in a few cases. On the other hand, due to the low density ratio(density of attached mass vs density of micro plate) the distributed mass caused very small changes on the resonant frequencies. However it induced distinct changes to the most mode shapes, especially in high modes.

\section{Comparison of results}

There are very few papers that discuss the dynamics of a distributive mass-loaded plate in a fluid. In order to verify the present method, the numerical results for plates without mass attachment vibrating in fluid are obtained to compare with the published results. An experimental dataset suitable for this comparison is the one published by Lindholm (1965) for the submerged cantilever plates(Lindholm et al., 1965). The dimensions and properties of the cantilever plate used here for comparison are: $L_{a}=L_{b}=8 i n c h, \rho_{p}=7.324 \times 10^{-4} l b / i n^{3}, E=30 \times 10^{6} \mathrm{psi}, \nu=0.3$. Table 1 compares the first five natural frequenies for this plate in vacuo and in water respectively.

\section{Insert Table 1}

The results of Table 1 show close agreement between the method of this paper and Lindholm's measurements. However quite large differences exist in the first two wet mode frequencies, as the plate used in our model is surrounded by an infinite baffle plane, while the plate of Lindolm's approach is resting on a free surface.

\section{Insert Table 2}

The comparison of NAVMI factors for simple-supported and clamped plates between the present method and Kwak's results(read from his data plot)(Kwak, 1996) are listed in Table 2. The aspect ratio $\varepsilon$ here is equal to 1 . It can be seen that this method can accurately calculate the NAVMI factors and therefore the natural frequencies of the submerged plates. 


\section{Conclusion}

This paper presents an analytical solution for the dynamic system of fluid-loaded plates with distributed mass attachment based on Lamb's model and the RayleighRitz energy method. Pierce's integral technique is used to overcome the difficulty of evaluating the effects of fluid loading. The numerical results calculated using the novel methodology are compared with Lindholm's experimental results and Kwak's NAVMI based results and show close agreement. It is concluded that the methodology proposed by this paper can be widely applied to most boundary conditions of rectangular plates, provided that the mode shape functions are known. It is the first analytical solution to this problem, taking into consideration both fluid loading and distributive mass loading effects. Both of these factors are important in micro-biosensor design using microstructures. Numerical results of plate resonant frequencies and mode shapes under different loading cases are presented. The novel methodology and the dynamical characteristics of microplates derived can be applied in the design of microplate based biosensing systems.

\section{Acknowledgment}

The authors wish to acknowledge funding support for this research from EPSRC and in particularly would like to thank Dr. W. R. Graham of the University of Cambridge for his suggestion on the integral evaluation.

\section{References}

C. Atkinsona and M. Manrique de Lara. The frequency response of a rectangular cantilever plate vibrating in a viscous fluid. Journal of Sound and Vibration, 300 (1):352-367, 2007.

Y.M. Chang and P. Leehey. Acoustic impedance of rectangular panels. Journal of Sound and Vibration, 64(2):243-256, 1996.

W.R. Graham. High-frequency vibration and acoustic radiation of fluid-loaded plates. Philosophical Transactions of the Royal Society, Series A, 352(1698): 1-43, 1995.

W.R. Graham. Analytical approximations for the modal acoustic impedances of simply supported rectangular plates. Journal of the Acoustical Society of America, 12(2):719-730, 2007.

J. E. Greenspon. Vibrations of cross-stiffened and sandwich plates with application to underwater sound radiators. The Journal of the Acoustical Society of America, 33(11):1485-1497, 1961.

M. C. Junger and D. Feit. Sound, Structures and Their Interaction. The MIT Press, Cambridge, Massachusetts, 1972.

O. Kopmaz and S. Telli. Free vibrations of a rectangular plate carrying a distributed mass. Journal of Sound and Vibration, 251(1):323-40, 2002. 
M.K. Kwak. Vibration of circular plates in contact with water. Journal of Applied Mechanics, 58(2):480-483, 1991.

M.K. Kwak. Vibration of circular membranes in contact with water. Journal of Sound and Vibration, 178(5):688-690, 1994.

M.K. Kwak. Hydroelastic vibration of rectangular plates. Journal of Applied Mechanics, 63(1):110-115, 1996.

H. Lamb. On the vibrations of an elastic plate in contact with water. Proceedings of the Royal Society of London, 98(A):205-216, 1921.

M. Lax. The effect of radiation on the vibrations of a circular diaphragm. Journal of the Acoustical Society of America, 16:5-13, 1944.

W. Leissa. Vibration of Plates. Scientific and Technical Information Division, National Aeronautics and Space Administration, US, 1969.

C. Liang, C. Liao, Y. Tai, and W. Lai. The free vibration analysis of submerged cantilever plates. Ocean Engineering, 28(9):1225-1245, 2001.

U.S. Lindholm, D.D. Kana, W.H. Chu, and H.N. Abramson. Elastic vibration characteristics of cantilever plates in water. Journal of Ship Research, 9:11-22, 1965.

W. K. Meyerhoff. Added masses of thin rectangular plates calculated from potential theory. Journal of Ship Research, 14:100-111, 1970.

Liviu Nicu and C. Ayela. Micromachined piezoelectric membranes with high nominal quality factors in newtonian liquid media: A lamb's model validation at the microscale. Sensors and Actuators B (Chemical), 123(2):860-868, 2007.

A.D. Pierce, R.O. Cleveland, and M. Zampolli. Radiation impedance matrices for rectangular interfaces within rigid baffles: Calculation methodology and applications. Journal of the Acoustical Society of America, 111(2):672-684, 2002.

R. Szilard. Theory and analysis of plates, classical and numerical methods. PrenticeHall, New Jersey, 1974.

W.O. Wong. The effects of distributed mass loading on plate vibration behavior. Journal of Sound and Vibration, 253(3):577-83, 2002.

F. Zhu. Rayleigh quotients for coupled free vibrations. Journal of Sound and Vibration, 171(5):641-649, 1994.

Article submitted to Royal Society 
Vibration analysis of submerged micro rectangular plates with distributed mass loading 13

Table 1. Comparison of natural frequencies $(\mathrm{Hz})$ of a cantilever plate submerged in fluid with Lindholm's experimental results

\begin{tabular}{ccccc}
\hline & \multicolumn{2}{c}{ In Vacuo } & \multicolumn{2}{c}{ In Water } \\
\hline Modes & Lindholm & Present & Lindholm & Present \\
\hline 1 & 99.5 & 99.3 & 51.4 & 45.0 \\
2 & 243.0 & 243.5 & 154.0 & 144.8 \\
3 & 610.0 & 622.5 & 355.0 & 358.7 \\
4 & 782.0 & 769.2 & 534.0 & 511.4 \\
5 & 887.0 & 898.2 & 585.0 & 590.9 \\
\hline
\end{tabular}

Table 2. Comparison of NAVMI factors of simple-supported and clamped plates with Kwak's results

\begin{tabular}{ccccc}
\hline & \multicolumn{2}{c}{ S-S-S-S } & \multicolumn{2}{c}{ C-C-C-C } \\
\hline Modes & Kwak & Present & Kwak & Present \\
\hline 1,1 & 0.41 & 0.41 & 0.35 & 0.3484 \\
$1,2(2,1)$ & 0.18 & 0.1823 & 0.16 & 0.1609 \\
2,2 & 0.13 & 0.128 & 0.12 & 0.1182 \\
\hline \multicolumn{5}{r}{} \\
& & & \\
$\overline{\overline{\bar{F}}}$ & & & $\overline{\overline{ }}$
\end{tabular}

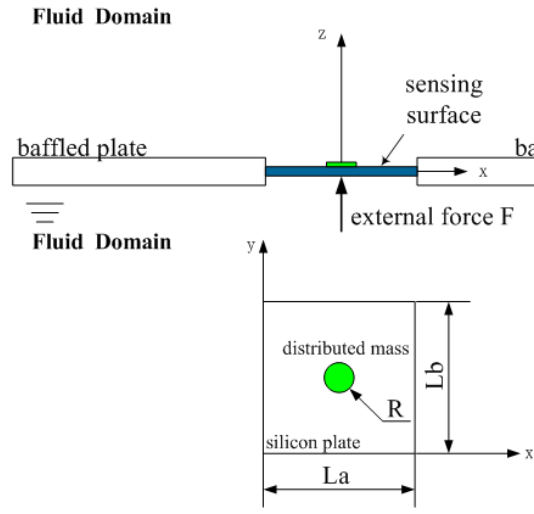

Figure 1. Schematic Diagram of Microplate Model 


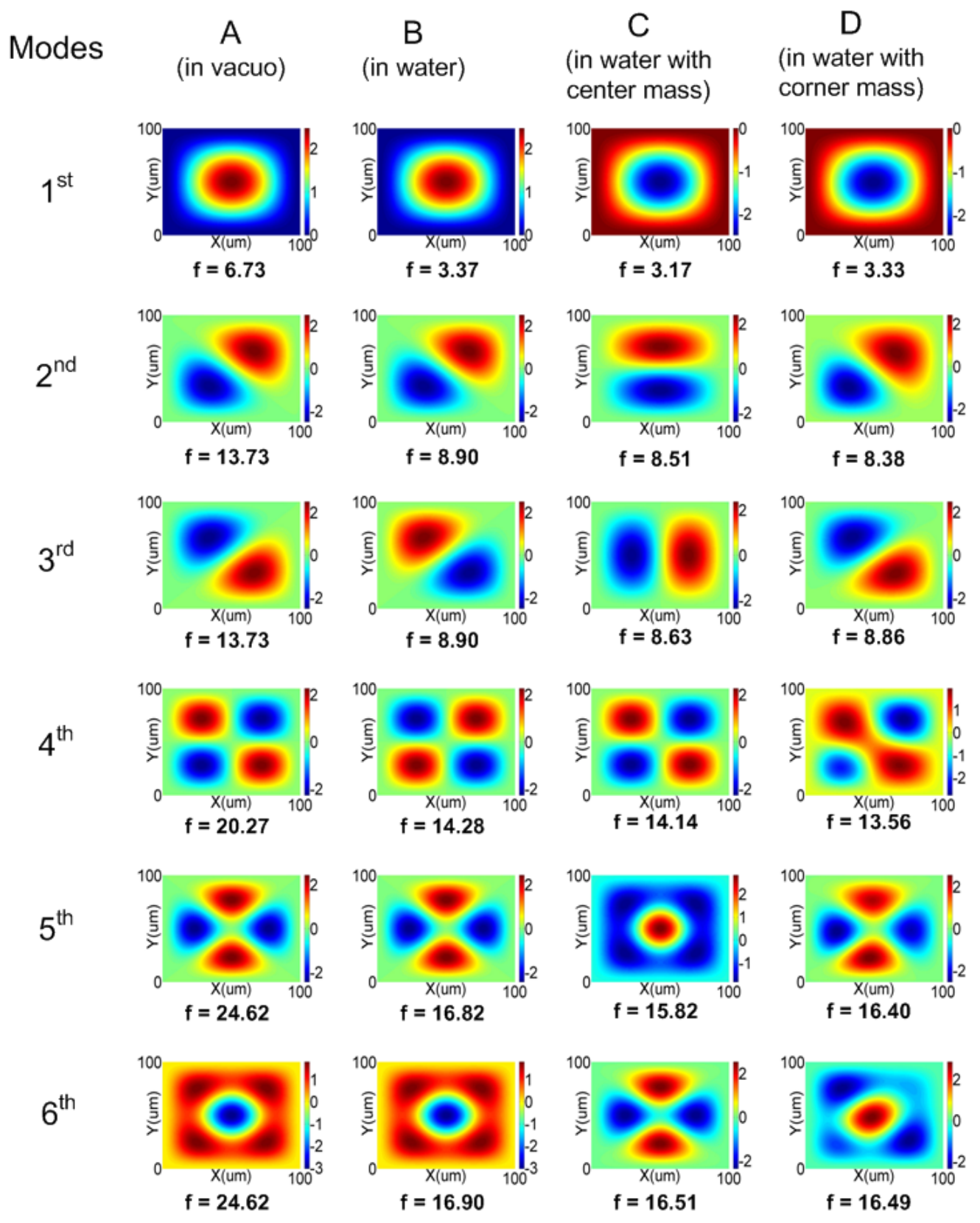

Figure 2. C-C-C-C Plate Resonant Frequencies(MHz) and Mode Shpaes 


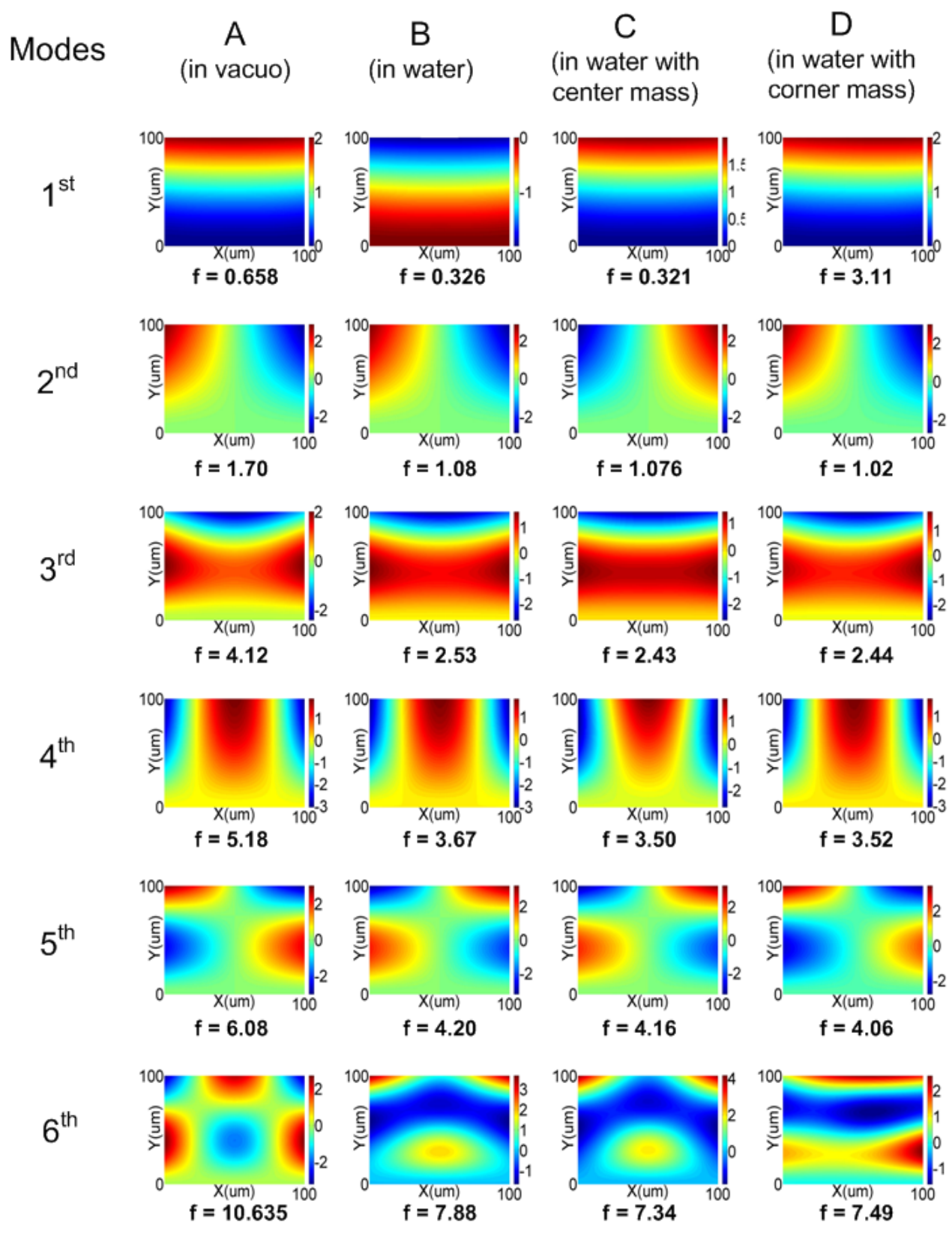

Figure 3. C-F-F-F Plate Resonant Frequencies(MHz) and Mode Shpaes 
Modes

A

(in vacuo)

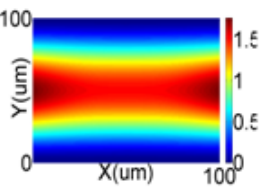

$f=4.18$

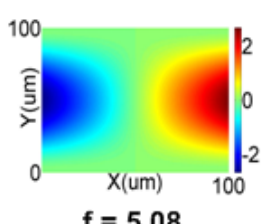

$f=5.08$

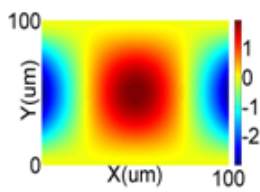

$f=8.38$

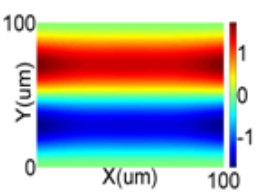

$f=11.53$

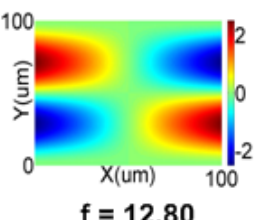

$f=12.80$

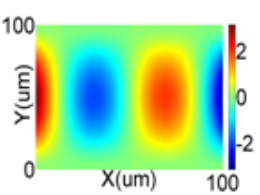

$f=15.18$
B

(in water)

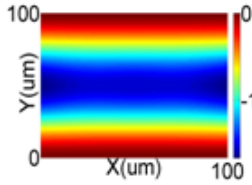

$f=1.98$
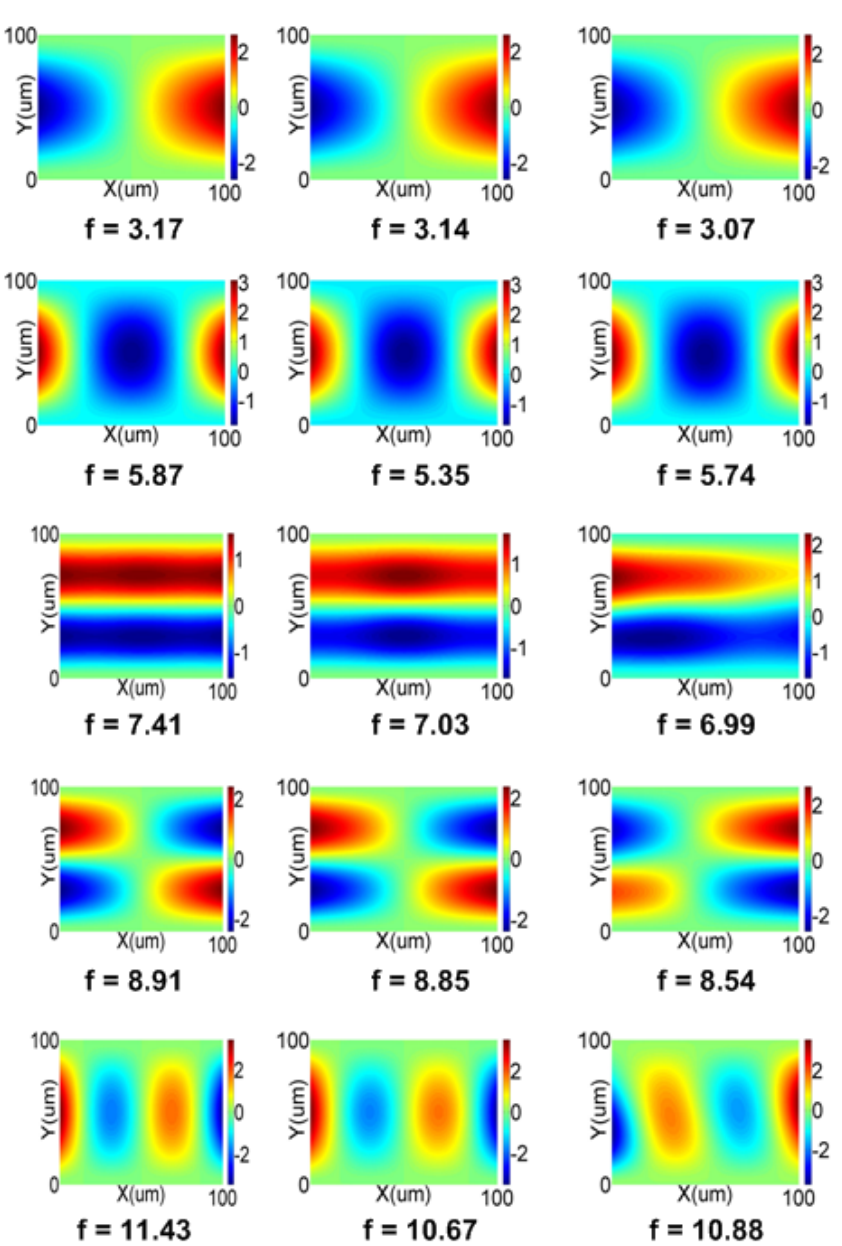

(in water with

corner mass)

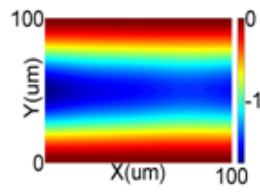

$f=1.94$
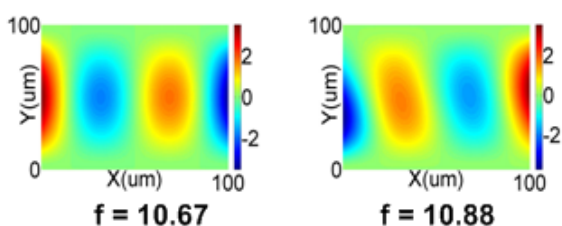

Figure 4. C-F-C-F Plate Resonant Frequencies(MHz) and Mode Shpaes 